DOI: $10.19195 / 0137-1169.38 .7$

\title{
Das Prinzip der Objektivität in akademischen Texten: Modalisierende Signale im deutsch-polnischen Kontrast
}

\section{Einführende Bemerkungen}

Der Beitrag ${ }^{1}$ befasst sich mit modalisierenden Textmarkern im akademischen Diskurs, insbesondere in studentischen Abschlussarbeiten. Modalisierende Marker, um die es sich im Weiteren handelt, sind Sprachmittel, die in irgendeiner Weise den Mitteilungssachverhalt aus der Perspektive des sprechenden Ichs qualifizieren und zu einem bewusst beabsichtigten kommunikativen Effekt (z.B. epistemische Beteuerung, Bekräftigung des eigenen Standpunktes vs. Distanzierung) beitragen. ${ }^{2}$ Die modalisierenden Marker, zu denen außer den Verbmodi auch lexikalische Mittel unterschiedlicher Art und Komplexität ${ }^{3}$ gehören, dienen also

\footnotetext{
${ }^{1}$ Die Arbeit ist entstanden im Rahmen des internationalen Forschungsprojekts INTERDISKURS (Interkulturelle Diskursforschung. Vergleichende Studien zur Textorganisation, zu den Formulierungsroutinen und deren Erwerbsphasen in den deutschen und polnischen studentischen Arbeiten), das aus Mitteln des NCN [dt. Nationales Zentrum für Wissenschaft] finanziert wird (Nummer der Entscheidung DEC-2013/08/M/HS2/00044). Der deutsche Teil des Projekts wird gefördert durch die Deutsch-Polnische Wissenschaftsstiftung (DPWS) (Projekt 2014-19).

${ }^{2}$ Davon zu unterscheiden sind die sog. verfasserreferentiellen Marker wie etwa meiner Meinung nach, meiner Ansicht nach usw., auf die hier (außer im Anhang) nicht speziell eingegangen wird. Interessierte verweise ich auf Schönherr 2017.

${ }^{3}$ Ein solches Herangehen schmälert in keiner Weise die Tatsache, dass Modalität primär als verbale Kategorie aufzufassen ist. Sie ist genuin in der morphologischen Ebene der Verbmodi beheimatet und dichotomisch organisiert: Während die konjunktivischen und imperativischen Modi statusmäßig als markierte Einheiten gelten, d.h. Einheiten mit maximal ausgeprägtem Sprecherbezug, verhält sich der Indikativ gegenüber dem Sprecherbezug neutral. Da auch nichtverbale Sprachmittel (wie etwa Modalpartikeln) den Sprecherbezug kodieren können, wird die Kategorie der Modalität auch auf andere
} 
dem Sprecher-Ich dazu, die mitgeteilten Informationen subjektiv zu beurteilen, individuell zu kommentieren oder für bestimmte Zwecke zu instrumentalisieren (Köller 1995:42).

Da die Signalisierung der subjektiven Haltung in wissenschaftlichen Texten oft als grundlegendes Merkmal der argumentativen Textkompetenz aufgefasst wird (vgl. Gätje/Rezat/Steinhoff 2012:125), gewinnt das Phänomen in der gegenwärtigen Forschungslandschaft kontinuierlich an Bedeutung, und zwar vor allem seitens der Schreibforschung und Schreibdidaktik. Dennoch findet eine Auseinandersetzung mit dem Thema bislang in erster Linie innerhalb der intralingualen Sprachforschung, also im Bereich der jeweiligen Muttersprache statt (vgl. z.B. Steinhoff 2007). Vergleichende Studien, die die Entwicklung argumentativer Schreibfähigkeiten konfrontativ in unterschiedlichen Sprachsystemen untersuchen, gibt es zwar nur vereinzelt, dafür sind sie aber sehr ertragreich. ${ }^{4}$ Demgegenüber sind Studien, deren Schwerpunkt auf der Analyse modalisierender Signale liegt, eher marginal, ganz zu schweigen von derartigen Untersuchungen zur fremdsprachlichen Textproduktion im akademischen Bereich. Das Schließen der Forschungslücken in diesem Bereich ist aber nicht die einzige Motivation zur wissenschaftlichen Auseinandersetzung mit dieser Thematik. Die Konfrontation polnischer Deutschlernender mit Textsorten aus der Wissenschaftsdomäne offenbart ein ganzes Spektrum an sprachlichen Defiziten, die in schriftlichen Studententexten besonders auffällig sind. Daher geht es bei der Untersuchung auch darum, diese Schwierigkeiten zu beschreiben und zu überprüfen, inwieweit sie in einem bewussten Lernprozess eliminiert werden können.

Die Frage, in welchem Ausmaß der Verfasser seine Präsenz und somit seine Subjektivität in wissenschaftlichen Texten signalisieren darf, ist ein seit Jahren intensiv diskutiertes Thema (vgl. u.a. Weinrich 1989, Kretzenbacher 1995, Gauger 1995, Olszewska 2016). Schon die Vielfalt der für ein und dasselbe Phänomen entwickelten Begriffe beweist, dass es in der Wissenschaft verschiedene - subjektiv geprägte - Perspektivierungsmöglichkeiten gibt. ${ }^{5}$ In jedem Akt der Darstellung von Phänomenen vollzieht sich nämlich nicht nur eine „objektorientierte unterschiedliche Sachakzentuierung der Phänomene“ (Köller 2004:330), sondern

Sprachentitäten ausgedehnt. So gesehen hat sie nicht nur den Status einer grammatischen Kategorie, sondern auch einer lexikalisch-grammatischen oder gar einer kommunikativ-pragmatischen.

${ }^{4}$ Es handelt sich u.a. um Arbeiten von Eßer 1997, Hufeisen 2002 und Kaiser 2002, deren Hauptaugenmerk sich auf den Aspekt der kulturspezifischen Textgenerierung konzentriert, im Besonderen im akademischen DaF-Bereich. Sie beweisen, dass das Schreiben in der Fremdsprache vielfach durch Interferenzen aus der Muttersprache erschwert ist, was sich z.B. in der Übertragung der internalisierten Textschemata oder Schreibstrategien auf die fremdsprachigen Textprodukte äußert.

5 Das ist übrigens nicht nur in formalisierten Fachsprachen wie der Sprache der Wissenschaft so. Für emotional relevante Sachbereiche wie Sterben oder Sexualität werden ganze Inventare von Begrifflichkeiten, darunter auch Metaphern, entwickelt, um die zu akzentuierenden Inhalte sprachlich wiederzugeben (vgl. Köller 2004:330). 
gleichzeitig eine subjektorientierte Interpretation derselben, die stets unter einem bestimmten Gesichtspunkt, ja von einer bestimmten (geistigen) Position des einzelnen Forschers aus erfolgt. Durch die Wahl der Wahrnehmungsperspektive bestimmt der Forscher, wie er die Sache sehen will, welche Aspekte in den Vordergrund treten, welche dagegen unbeachtet bleiben sollen etc. Dass es also in der Wissenschaft (aber auch in anderen Sachbereichen) unterschiedliche Stile der Weltwahrnehmung gibt, die sich zum Teil verschränken, zum Teil aber stark voneinander abweichen, ist ein völlig legitimes, anthropologisches Phänomen, wovon vielfach sowohl die - wie ich sie nennen möchte - „hochkarätige“ als auch die „studentische“ Wissenschaft Gebrauch machen. Das zweite Argument, warum man die Präsenz des Verfassers im wissenschaftlichen Diskurs nicht ausschließen kann, ist der rhetorische Charakter eines jeden Textes, darunter auch des wissenschaftlichen (vgl. Dönninghaus 2005:558). Dieser hat - ähnlich wie die bereits in der antiken Rhetoriktradition formulierten Anforderungen - nicht nur eine rein informative Funktion (docere), sondern erfüllt zugleich (wenn auch in einem deutlich geringeren Ausmaß) die Funktion des Unterhaltens (delectare) und dient nicht zuletzt zur Beeinflussung potentieller Textrezipienten (movere). Mithin erfüllen wissenschaftliche Texte einerseits die Funktion der Informationsvermittlung, andererseits haben sie einen persuasiven Charakter, der sich nicht einfach aus dem Inhalt des Textes ergibt, sondern aufgrund der Relation zwischen dem Textverfasser und dem Textrezipienten zustande kommt: Denn je mehr Einfluss der Autor in seinem Text auf den Rezipienten ausüben will, umso mehr modalisierende Ausdrücke werden darin vorkommen und umso höher wird der Modalisierungsgrad des Textes sein. Das in der - vor allem älteren - Fachliteratur oft geforderte Ich-Verbot (Weinrich 1989), das von vielen für ein übergeordnetes Kriterium des wissenschaftlichen Stils gehalten wird, lässt sich daher paradoxerweise kaum einhalten und gilt insofern eher als richtungsweisendes Ideal denn als zu erreichendes Ziel. Wieviel Subjektivität in einen Text hineingebracht wird, ist nicht zuletzt auch vom soziokulturellen Hintergrund des wissenschaftlichen Autors abhängig und daher für eine vergleichende Studie wie diese besonders interessant.

\section{Schwerpunkte der Untersuchung}

Im Unterschied zum deutschsprachigen Hochschulwesen lässt sich in der polnischen Hochschuldidaktik eine eher geringe Schreibfrequenz von DaF-Studenten und eine daraus resultierende eingeschränkte Schreibpraxis verzeichnen (vgl. Zuchewicz 1997 und 2001). Die Hauptgründe dafür sind einerseits in der Organisation des Studiums selbst zu suchen. Da die Erbringung der Qualifikationsnachweise in den meisten Fällen auf mündlichem Wege erfolgt, entfällt größtenteils eine reguläre wissenschaftliche Schreibübung, sodass die Vorbereitung der 
Abschlussarbeit am Ende der Studienzeit für viele Studierende eine erste große Herausforderung im Bereich des akademischen Schreibens darstellt. Der zweite Grund ist darin zu suchen, dass die meisten Schreibkurse relativ wenig aufgefächerte Schreibanlässe bieten. Häufig handelt es sich um Gebrauchstextsorten wie Briefe, Dialoge oder Zusammenfassungen. Demgegenüber werden wissenschaftstypische Textsorten wie Seminararbeiten oder Kommentare selten geübt. All das führt dazu, dass das wissenschaftliche Schreiben(lernen) in der Fremdsprache Deutsch mühsam verläuft und mit für das fremdsprachliche Umfeld typischen, manchmal kaum überwindbaren Schwierigkeiten (imitatives Schreiben, Reproduktion von Mustertexten etc.) verbunden ist. Auch bei im Laufe der Studienzeit zunehmender Vertrautheit mit Schreibregeln und Textsortenkonventionen erweist sich der produktive Umgang mit Texten für viele Studierende als eine nicht zu überwindbare Barriere, was entweder das Problem der fristgerechten Fertigstellung der Abschlussarbeit oder - im Extremfall - den Studienabbruch zur Folge hat. Ausgehend von dem übergeordneten Lernziel der Fremdsprachendidaktik, die auf dem Prinzip beruht, dass Sprachenlernen gleichzeitig auch Schreibenlernen bedeutet, sollte dem Prozess der fremdsprachlichen Textproduktion deutlich mehr Aufmerksamkeit geschenkt werden. Dabei soll es allerdings nicht bzw. nicht nur um die Vermittlung informationsbezogener Kenntnisse hinsichtlich der Konzipierung wissenschaftlicher Texte ${ }^{6}$ gehen, sondern um die Vermittlung konkreter Textkompetenzen. Eine davon ist ohne Zweifel die Kompetenz der Modalisierung. Gegenstand der Untersuchung ist daher die Analyse des Erwerbs und des Gebrauchs modalisierender Signale in wissenschaftlichen Studententexten. Insgesamt verfolgt die vorliegende Studie drei Zielsetzungen: Es soll gezeigt werden, (i) welche kulturspezifischen Schreibkonventionen den Gebrauch von modalisierenden Signalen beeinflussen, (ii) welche sprachlichen Schwierigkeiten und Strategien bei der Verwendung von modalisierenden Signalen auftreten, und (iii) wie der Schreibprozess in didaktischer Sicht im Hinblick auf deren Gebrauch optimiert werden kann. Schon im Vorfeld der Untersuchungen konnte beobachtet werden, dass das Schreiben in der Fremdsprache - abgesehen vom Individualstil einzelner Schreibender - zwischen dem eigenen und fremden Kulturparadigma oszilliert, die je nach Sprachgemeinschaft unterschiedliche Normvorstellungen hervorbringen. Aus diesem Grund darf sich die Untersuchung nicht nur der fremdsprachigen, sondern auch der fremdkulturellen Interferenzen durchaus als lohnend erweisen. Hinzu kommt, dass die Verwendung der modalisierenden Si-

\footnotetext{
${ }^{6}$ Es wäre wohl treffender, hierbei von einer Form der „,vorwissenschaftlichen“ oder „präwissenschaftlichen" Textproduktion zu sprechen, denn studentische Elaborate (sei es Seminar- oder Abschlussarbeiten) sind im Unterschied zu wissenschaftlichen Texten nicht darauf ausgerichtet, neue Wissensbestände zu erschließen bzw. publiziert zu werden. Sie dienen gewissermaßen als Übungstexte, deren Ziel es ist, sich in den wissenschaftlichen Stil einzuüben (learning to write) und auf diese Weise Fachwissen zu erwerben (writing to learn).
} 
gnale in erster Linie nicht auf den Stand der angeeigneten Sprachkenntnisse in der Fremdsprache zurückzuführen ist, sondern mit den jeweiligen sprachlichen und kulturellen Konventionen in Zusammenhang steht.

\section{Korpus und Untersuchungsmethode}

Grundlage für die empirische Analyse bildet ein Textkorpus, zusammengesetzt aus studentischen Abschlussarbeiten, die von polnischen Germanistikstudenten verfasst wurden und die Zeitspanne von 2008 bis 2013 erfassen. Es handelt sich um Texte aus der Domäne der germanistischen Sprach- und Literaturwissenschaft. Je nach Hauptausrichtung (Sprachwissenschaft versus Literaturwissenschaft) sind es Literaturstudien oder vergleichende bzw. historische Analysen. ${ }^{7}$ Dabei weisen sie strukturell gesehen eine Zweiteilung auf. Während der erste Teil in der Regel einen theoretischen Hintergrund bildet, der neben einer summarischen Darstellung der wichtigsten Theorien zum behandelten Thema auch Hinweise zum methodischen Analyseverfahren enthält, ist der zweite Teil der Arbeit mehr oder minder empirisch ausgerichtet. Dieser Struktur entsprechend sollen die Abschlussarbeiten - zumindest vom Prinzip her - argumentativ relevante Formulierungsmuster beinhalten und darauf abzielen, sich mit einem konkreten Problem wissenschaftlich auseinanderzusetzen, also die Aspekte des aktuellen Forschungsstandes zu diskutieren und sie kritisch zu bewerten. Dies setzt voraus, dass über das Zitieren aus der Sekundärliteratur hinausgehende Aussagen getroffen, Fragen gestellt und Argumente abgewogen werden müssen. Dass es Abweichungen von diesem (ideell anmutenden) Modell gibt, gilt als selbstverständlich.

Daran anschließend werden auch polonistische Studentenarbeiten aus dem muttersprachigen Kontrastkorpus herangezogen. Im Hintergrund wird auch auf deutschsprachige wissenschaftliche Expertentexte ${ }^{8}$ aus dem Kontrollkorpus Bezug genommen. Auf diese Weise soll geprüft werden, welche Ähnlichkeiten und Unterschiede beim Gebrauch von modalisierenden Markern in studentischen fremdsprachigen gegenüber muttersprachigen Arbeiten vorkommen sowie welche Interferenzen sich jeweils feststellen lassen. Andererseits sollen wissenschaftliche Texte von Schreibexperten veranschaulichen, inwieweit zwischen dem studentisch-akademi-

\footnotetext{
7 Bei der Abfassung von Abschlussarbeiten wird großer Wert auf eine möglichst hohe Eigeninitiative der Studierenden gelegt. Nichtsdestotrotz greifen die betreuenden Dozenten auf allen Etappen der Schreibbetreuung in den Textproduktionsprozess ein. Diese reicht - so unsere Erfahrung - von der Korrektur der sprachlichen Fehler bis hin zu inhaltsbezogenen bzw. sachlogischen Verbesserungsvorschlägen und Hinweisen zur Textgliederung. Was die Texte des hier zur Untersuchung vorliegenden Korpus anbelangt, so muss der Aspekt der Fehlermarkierung außer Acht bleiben, da nicht (mehr) feststellbar ist, wie viel Anteil der jeweilige Dozent am Entstehen der jeweiligen Arbeit hatte. 8 Es handelt sich um ausgewählte Kapitel aus Monographien und Aufsätzen anerkannter Sprachund Literaturwissenschaftler.
} 
schen Schreibduktus und dem wissenschaftlichen Stil par excellence ein (Miss-) Verhältnis bezüglich der Verwendung und Funktionalisierung von modalisierenden Signalen besteht. Eine Untersuchung der deutschen Studententexte wird hier nicht speziell unternommen, da derartige, zum Teil auch kontrastiv angelegte Studien (vgl. z.B. Kaiser 2002) bereits vorliegen. Insofern wird im Rahmen der vorliegenden Arbeit an die bereits vorhandenen Forschungsergebnisse angeknüpft.

Die Auswertung des Untersuchungskorpus von 30 studentischen Elaboraten hat insgesamt 171 Gebrauchsfälle der modalisierenden Mittel ergeben. Es hat sich gezeigt, dass nahezu jeder Einzelfall mit seiner singulären, durch die aktuelle Äußerungssituation bedingten Besonderheit einer separaten Behandlung bedarf. Dies ist allerdings im beschränkten Rahmen der vorliegenden Arbeit nicht möglich. Ebenfalls unmöglich ist die quantitative Erfassung aller im Korpus vorkommenden Belege. Die Speicherung der Belege verlief in der ersten Phase der Korpusrecherche systematisch: Alle gefundenen Belege wurden der Reihe nach in die Belegdatenbank aufgenommen. Eine solche Vorgehensweise sollte ursprünglich sicherstellen, dass keine der vorkommenden Formen unberücksichtigt bleibt. Erst in der späteren Phase der Korpusuntersuchung wurde der Fokus zunehmend auf untypische Verwendungskontexte gerichtet, in denen entweder ein höchst interessanter oder problematischer bzw. fehlerhafter Gebrauch von modal markierten Formulierungen vorliegt. Die Tatsache, dass einige Kontexte in die Analyse nicht mit einbezogen wurden, schmälert allerdings nicht die Aussagekraft der erzielten Ergebnisse. Viel wichtiger sind hier die ermittelte funktionale und formale Vielfalt der exzerpierten Formen sowie die Erkennung von Schwierigkeiten der Schreibenden bei deren Gebrauch, woraus wiederum wertvolle Rückschlüsse auf die bestehenden Lücken und Desiderata im didaktischen Prozess gewonnen werden können.

\section{Forschungsergebnisse}

Die durchgeführten Korpusanalysen haben, wie bereits oben erwähnt, eine Reihe von aufschlussreichen und didaktisch verwertbaren Erkenntnissen erbracht. Diese lassen sich wie folgt zusammenfassen:

1. Die exzerpierten Modalisierungssignale lassen sich sowohl in funktionaler als auch formaler Sicht beschreiben. Grob geschätzt können drei Funktionalbereiche erschlossen werden, in denen die modalisierenden Ausdrücke am häufigsten auftreten. Es sind (1) Markierungen eigener Einschätzungen bezüglich des vorgestellten Forschungsproblems, (2) Bewertungen der diskutierten Forschungsergebnisse bei der Herstellung von intertextuellen Bezügen und (3) die Bekräftigung der eigenen Argumentation bzw. die Abschwächung des Geltungsgrades einer (fremden) Aussage. Während die ersten zwei Bereiche eine natürliche Vorkommensdomäne modalisierender Ausdrücke darstellen, scheint der letztere Bereich 
weniger prädestiniert für die Verwendung von subjektiv-modalisierenden Ausdrücken zu sein, denn gerade bei Argumentationsstrategien sollen nicht subjektive Überzeugungen des Autors, sondern objektive und sachlich-rationale Argumente dargestellt werden. Aus formaler Sicht können wir festhalten, dass zur Wiedergabe sprecherbezogener Urteile einerseits evaluierende, andererseits epistemische Adverbien gebraucht werden. Während die epistemischen Kommentaradverbien geltungsbezogene Sprechereinstellungen markieren, die auf der Skala von sicher bis unsicher positioniert werden können, haben evaluierende Adverbien eine Kommentarfunktion. Anders als die epistemischen Modalisierungssignale, die den jeweiligen Sachverhalt hinsichtlich seiner Faktizität qualifizieren, geben evaluierende Adverbien eine beurteilende oder emotionale Reaktion des Schreibers auf einen Sacherhalt wieder, welche zwischen dem positiven (hoffentlich) und negativen Pol (leider) oszilliert. Darüber hinaus gibt es metakommunikative Einschätzungssätze und -syntagmen, die sich durch eine strukturelle Komplexität und ein problematisches Distributionsmuster auszeichnen.

2. Beim Vergleich germanistischer Arbeiten mit Texten aus dem polnischen Kontrastkorpus fällt auf, dass in den Letzteren sich ein völlig anderes Bild der exzerpierten Modalisierungssignale im Hinblick auf ihre Verwendung ergibt: Polnische Studententexte tendieren in der Regel zur Verwendung schlichter und kompakter Modalisierungssignale. Strukturell komplexe Modalisierungsausdrükke kommen hier sehr selten vor, während in germanistischen Arbeiten polnischer Studierender sich ein Hang zur übertriebenen Betonung der subjektiven Stellungnahme aufspüren lässt. Hinter diesen Unterschieden stehen nicht nur divergierende kommunikative Absichten (objektiver Sachstil versus subjektive Wertungen), sondern auch ein anderer Grad der Internalisierung der wissenschaftstypischen Schreibpräferenzen in der jeweiligen Sprache.

3. Der vielerorts unbeholfene Stil der polnischen Germanistikstudenten hinsichtlich der Verwendung von modalisierenden Signalen ist nicht nur darauf zurückzuführen, dass sie in einer fremden Sprache schreiben, sondern auch darauf, dass sie sich in einer fremden Wissenschaftssprache angemessen ausdrücken müssen. Viele der fehlerhaften Formulierungen sind daher nicht allein mangels der schwach ausgeprägten Grammatik-, Text- oder Sachkompetenzen entstanden. Es sind dabei auch konventionalisierte bzw. kulturbedingte Interferenzen zu nennen, die das wissenschaftliche Schreiben in Deutsch als Fremdsprache ungünstig beeinflussen und zu Formulierungsfehlern führen.

\section{Diskussion der Forschungsergebnisse}

Ad. 1. Die modalisierenden (evaluativen und epistemischen) Kommentaradverbien treten in den untersuchten studentischen Arbeiten überraschenderweise selten auf. Es gibt Texte, die keine oder nur einzelne Kommentaradverbien enthalten, 
dafür aber ein reichlich ausgebautes Repertoire an epistemischen Verben des Denkens oder sonstigen subjektiv geprägten Formulierungen. Es fällt zudem auf, dass die Adverbien (wie überhaupt die meisten modalisierenden Signale) tendenziell im zweiten, also empirischen Teil der Arbeiten vorkommen. Die theoretisch ausgerichteten Kapitel bleiben häufig in dieser Hinsicht unmarkiert und zeigen typische Kennzeichen einer kompilatorischen Darstellung der wichtigsten Theorieansätze zum behandelten Thema. Die am häufigsten verwendeten Adverbien sind sicher(lich) und wahrscheinlich. Sie werden verwendet, um den Geltungsgrad der Aussagen zu modifizieren, so auch in folgenden Textpassagen:

Der Lautwandel betrifft sowohl Konsonanten als auch Vokale, die sich im Laufe der Jahre wesentlich verändert haben. Es ist schwer, die Ursachen für den Lautwandel zu zeigen. Sicher ist, dass es bestimmt viele Faktoren gab, die den Lautwandel beeinflusst haben.

Wie fühlt sich jetzt der Schüler? Welchen Einfluss kann das Gesicht des Lehrers auf ihn haben? Wahrscheinlich fühlt er sich unsicher. Er weiß nicht, ob seine Antwort richtig oder falsch ist. [...] Wie sieht jetzt der Lehrer aus? Sein Gesicht lacht, wahrscheinlich hört er sich die Äußerung des Schülers an.

Obwohl die Kommentaradverbien in der deutschen Sprache als prototypische Mittel zur Markierung sprecherbezogener Stellungnahmen bereitgestellt werden, drängt sich angesichts ihrer niedrigen Vorkommensfrequenz die Frage auf, warum sich dieses Kodierungsverfahren keiner großen Beliebtheit bei der Anwendung von modalisierenden Strategien erfreut. Eine Antwort ist bereits in den Textauszügen selbst angelegt. Es lässt sich hier nämlich eine gewisse Unsicherheit der Schreibenden beim Gebrauch von Kommentaradverbien aufspüren, so z.B. schon im ersten Textbeispiel, wo sich zwei funktionsähnliche Kommentaradverbien (sicher und bestimmt) überschneiden und wo es dadurch zur redundanten Markierung des Sprecherbezugs kommt. Das eigentliche Problem beim Gebrauch von Kommentaradverbien liegt darin, dass die Schreibenden sich oft über die Wirkung dieser sprachlichen Formen nicht im Klaren sind und den Schwerpunkt bei der Textgenerierung eher auf eine gelungene, ausdrucksseitig korrekte Formulierung als auf die inhaltliche Richtigkeit derselben legen. Es ist daher davon auszugehen, dass das Kommentaradverb sicher im konkreten Fall nicht inhaltlich bzw. funktional motiviert ist, d.h. nicht zur Markierung der eigenen Meinung des Schreibers, sondern eher zur Kontrastierung des im vorausgehenden Satz dargestellten Aussageinhalts gesetzt wird (Es ist schwer... vs. Sicher ist...). Mit anderen Worten, es wird nicht als Modalisierungsmittel, sondern als stilistisches Mittel verwendet, in dessen Folge zwar ein interessantes Syntaxmuster (Kontrast, Chiasmus), auf der Inhaltsseite aber ein, wenn nicht ganz expliziter, so doch erkennbarer Lapsus entstanden ist. 
Im zweiten Beispiel fällt auf, dass der Autor sich damit schwertut, durchgehend einen sachlichen Schreibstil einzuhalten. Er ist bemüht, sich sprachlich von einer rhetorischen bzw. poetischen Ausdrucksweise zu distanzieren. Für die Darstellung seiner Ausführungen wird die Frage-Antwort-Strategie gewählt, die allerdings zweimal in der Folge vorkommt und daher als störend empfunden wird. Das Adverb wahrscheinlich wird ebenfalls zweimal verwendet, jeweils als Teil der Antwort auf die im vorausgehenden Satz gestellte Frage. Während gegen den ersten Gebrauch des Kommentaradverbs nichts einzuwenden ist, scheint seine Verwendung im zweiten Fall nicht ganz treffend zu sein. Der Autor bezieht sich hier auf eine evidente Tatsache, d.h. auf den Gesichtsausdruck der beschriebenen Person (sein Gesicht lacht), der für ihn als Quelle seiner Vermutungen fungiert. Wir haben hier also eine prototypische Situation einer Evidentialität vorliegen, und nicht eine der „reinen Epistemizität“. Bei epistemischen Urteilen bezieht sich der Sprecher bekanntlich nur auf seine ,inneren“ Überzeugungen, während bei evidentiellen Urteilen diese sich auf unterschiedliche, meist fremde Quellen (Hörensagen, Bilder, (Kon-)Texte usw.) stützen können (vgl. z.B. Kotin/Schönherr 2012). Viel treffender wäre es daher, anstatt des genuin epistemischen Adverbs wahrscheinlich ein evidentielles Adverb, etwa offensichtlich zu verwenden. Welche Erkenntnisse liefern uns diese Teilanalysen? Sie zeugen vor allem davon, dass die Studierenden über ein nicht breit genug gefächertes grammatisches Wissen verfügen und in der Überzeugung schreiben, dass sie richtig schreiben, ohne über das Geschriebene im Kontext der grammatischen Korrektheit nachdenken zu können.

Was die evaluativen Kommentaradverbien angeht, so sind sie im untersuchten Textkorpus ebenfalls selten belegt und grundsätzlich auf das Wort leider beschränkt, das zuweilen im Rahmen der Textsequenzen mit textorganisierenden Hinweisen vorkommt:

In dem theoretischen Teil habe ich den Kommunikationsprozess an verschiedenen Modellen beschrieben, leider ist dieses Thema sehr ausführlich, deshalb habe ich mich nur auf paar Modelle beschränkt.

Was hier zum Problem wird, ist das Bestreben des Autors, seine Meinung zum Umfang des behandelten Themengebiets abzugeben. Dass das behandelte Thema umfang- und facettenreich ist, ist aus wissenschaftlicher Sicht gerade als positiv zu bewerten und zeugt von der Intensität und Vielseitigkeit der Forschungen, die auf diesem Feld betrieben werden. Es ist also die noch nicht ausgebildete Fähigkeit des Schreibers, wissenschaftliche Probleme sachgemäß beurteilen zu können, über die er stolpert. Stattdessen beurteilt er die Problemlage aus seinem (engen) Erfahrungsraum heraus, was im Endeffekt dazu führt, dass seine Interpretation in die falsche Richtung geht. 
Demgegenüber weisen modalisierende Signale, bei denen eine strukturelle Komplexität vorliegt, ein relativ systematisches Gebrauchsmuster auf. Dabei handelt es sich einerseits um metakommunikative Einschätzungssätze (wie etwa es ist kaum zu bezweifeln, dass...), andererseits um relativ verfestigte Syntagmen, darunter Kollokationen (es unterliegt keinem Zweifel) und Funktionsverbgefüge (es steht außer Zweifel). Es hat den Anschein, dass derartige Formen in erster Linie - wie aus den folgenden Beispielen ersichtlich - für literaturwissenschaftliche Arbeiten typisch sind und viel häufiger dort als in Texten mit sprachwissenschaftlicher Ausrichtung vorkommen:

Der heterodiegetische Erzähler präsentiert sehr gut die Einstellung des Vaters zu seinem Kind. Es steht außer Zweifel, dass diese Einstellung negativ ist, und dass sich diese im Fortgang der Zeit noch verschlechtert [...].

Dass Leo mit seiner einzigen Tochter nicht in Kontakt bleiben will, unterliegt keinem Zweifel $[\ldots]$.

Es unterliegt keinem Zweifel, dass der Erzähler den Roman mit dem SexSkandal der 1950er Jahre einsetzt.

Nach Martinet ist es also kaum zu bezweifeln, dass die Sprachentwicklung durch die Tendenz zur Ökonomie bestimmt wird.

Es ist unbestritten, dass die Sprache ein Phänomen ist, das dem ständigen Wandel unterliegt.

Es scheint Jule nicht mehr zu stören, dass Liz ihre emotionalen Bedürfnisse nicht befriedigt.

Eine für die Wissenschaftskommunikation besonders untypische Modalisierungstechnik findet sich in folgenden Textauszügen:

Von meinen subjektiven täglichen Beobachtungen aus ist kaum zu bezweifeln. dass der Unterschied in der Kommunikation zwischen den Lehrern und den Lehrerinnen sofort auffällt. Aber in dieser Phase wende ich mich lieber konkreten Beispielen aus dem Lehrerzimmer zu.

Für mich und meine subjektiven Einschätzungen der Situation besteht kein Zweifel daran, dass diese ,Wirklichkeit” sich für Frauen in der Regel als nachteilig erweist, da die als typisch weiblich festgelegten Merkmale auch in der heutigen Zeit insgesamt weniger gesellschaftliches Ansehen genießen als die männlichen.

Man sieht hier deutlich, dass die Prozeduren der Modalisierung dem wissenschaftlichen Stil weitgehend nicht entsprechen. Die ausgebauten Modalisierungsstrukturen erinnern eher an alltägliche mündliche Argumentationsstrategien, bei denen es bekanntlich auf aussagekräftige, strategische „High-Power-Formulierungen“ ankommt. 
Wie die Korpusanalyse zeigt, ist die Distribution modalisierender Sprachmittel in studentischen Texten manchmal an die sprachlichen Muster der Originalvorlage angelehnt, aus der der Autor Sachverhalte oder Anregungen für seinen Text schöpft. Wenn man sich jedoch die Texte etwas genauer anschaut, dann wird klar, dass die Autoren - auf der Originalvorlage aufbauend - sehr schnell selbst eigene Modalisierungstechniken und -präferenzen entwickeln, die sich z.B. in einer kumulativen Verwendung von bestimmten Formulierungsstrukturen äußert, vgl. die folgenden Auszüge aus einer Magisterarbeit:

Im Folgenden versuche ich also zu erläutern, welche Ansichten der Autoren zum Thema geschlechtstypisches Kommunikationsverhalten über die Jahre besondere Wirkung erzielt haben, wie sie in meinen Augen zu bewerten sind und wie die neuere Forschung hinsichtlich dieses Themas, auch im Vergleich zur älteren Forschung aussieht (S. 7).

Um Zusammenhänge zwischen dem Kommunikationsverhalten einer Person und ihrem Geschlecht zu ergründen, muss Geschlecht in meinen Augen zweifellos im Sinne von gender verstanden werden (S. 8).

In dieser Arbeit werde ich unter anderem einen zusätzlichen Einflussfaktor zutage treten lassen, der in meinen Augen zu Unrecht in sehr vielen Forschungen entweder ganz ausgelassen oder nur teils wahrgenommen wird (S. 8).

Für mich ist der Erklärungsansatz von Tannen zu sehr darauffokussiert, eine klare Differenz zwischen beiden Geschlechtern zu finden und diese dann auszuspielen bzw. sie sehr zu betonen. In meinen Augen liefert Tannen in ihrem Buch zu leichte Antworten auf schwierige Fragen und scheut gleichzeitig nicht vor großen Generalisierungen (S. 9/10).

Insgesamt ist in meinen Augen festzuhalten, dass das Forschungsfeld „,Geschlechtstypisches Kommunikationsverhalten" sich zu einem recht produktiven Forschungszweig entwickelt hat (S. 10).

Damit spreche ich auch einen wesentlichen Gesichtspunkt der Kommunikation an. Denn in meinen Augen kommt der nonverbalen Kommunikation im Kommunikationsprozess eine wichtige Rolle zu (S. 14).

Und so weiter im gesamten Text der Magisterarbeit.

Ad. 2. Im polnischen Kontrastkorpus, das insgesamt 20 Abschlussarbeiten aus dem Bereich der Bachelor- und Masterarbeiten umfasst, sind in überwiegender Zahl der Modalisierungskontexte Kommentaradverbien zu verzeichnen. Kreativen und komplexen modalisierenden Formulierungen begegnet man dagegen relativ selten. Im Vergleich zu germanistischen Arbeiten, in denen in vielen Kontexten ein überschwänglicher, ja poetischer Schreibstil feststellbar ist, haben wir es hier mit einem umgekehrten Gebrauchsmuster zu tun. Es überwiegen schlichte und kompakte Modalisierungsausdrücke: 
Koniński prawdopodobnie jest jednym z pedagogów, a zarazem kierownikiem, pracujacym $w$ pótinternacie przy ul. Nowolipki 25 zorganizowanym przez Cento [... wahrscheinlich ...].

Petna więc praca nad arcydzietem trwała nie pięć, ale tylko najprawdopodobniej od wiosny 1861 do jesieni 1862, czyli przez póttora roku [... höchstwahrscheinlich ....].

Ten fragment z cata pewnościq uświadamia nam, że Waldorffa interesowaty wszelkie informacje zwiazane z muzyka [... mit voller Sicherheit ...].

Najwięcej informacji o wydanych książkach znalazłam po roku 1990. Dziesięciolecie 1990-2000, to czas przemian nie tylko politycznych, ale także i spotecznych i kulturowych, które niewatpliwie przyczynity się do rozwoju duchowego społeczeństwa [... unzweifelhaft...].

Po co zatem ci ludzie przyszli na pogrzeb? Zapewne z obowiazku, $\underline{\text { może z cie- }}$ kawości [... gewiss ... vielleicht...].

Może wtaśnie owa nieskończoność jest tym nieznanym nam Końcem, który ludzie zwykli nazywać dusza, niebem, karma czy zaświatami [Vielleicht ...].

Ziemlich oft erscheinen auch modal-evidentiale Sprachmittel, mit denen der Sprecher die einer konkreten Quelle entnommene Information epistemisch relativiert:

Najważniejsze, bądź zapamiętane kobiety Marka Hłaski to: Wanda, kilka lat starsza kobieta z dzieckiem, z która chciat wziąc ślub jako młody chtopak [...], Hanna Golde, podobno największa i jedyna mitość Marka Htaski [...] [... angeblich ...].

Po ślubie z Aurelia z Szacsznajdrów Szabłowska w 1902 roku, Reymont wiódt ponoć stateczny żywot dosyć przykładnego matżonka [... angeblich ...].

Dzietu temu badacze zarzucaja jakoby byto poszerzeniem tylko idei utworu Poeta $i$ świat. Nie jest to jednak prawda, pisarz sam odpiera ten zarzut $w$ cytowanej już przedmowie stowami: [...] [... angeblich ...].

Fragment listu Marka do matki z dnia 14 sierpnia 1954 roku przedstawia jego wizerunek rzekomo niekochanego przez rodzicielke, bezbronnego i nie potrafiacego poradzić sobie z problemami, które sam na siebie ściagnąt [... angeblich ....].

Das Paradebeispiel für evaluative Markierungen ist das Modalwort niestety ,leider‘:

Ostatnia część tego rozdziału poświęcona będzie krośnieńskiemu pisarzowiAlfredowi Henschke Klabundowi, o którym niestety Krośnianie nie pamiętaja [... leider ...].

Neben Kommentaradverbien werden auch periphrastische Formen der Modalisierung benutzt: 
Wyrazy te nie figuruja w SPXVI, sa jednak obecne w Stowniku Lindego i Stowniku wileńskim. Nie możemy jednak być do końca pewni, czy wyrazy te powstały na pewno $w$ dobie oświecenie, mogły bowiem istnieć już wcześniej tylko w mowie potocznej, mogło również dojść do takiej sytuacji, iż teksty, które je zawieraty, zostaty zniszczone [Wir können uns jedoch nicht völlig sicher sein, ob...].

Mam nadzieje, że moja praca przyczyni się do poszerzenia spojrzenia na temat obrazów miłosnych $w$ twórczości tego XVIII wiecznego poety [... ich hoffe, wörtl.: ich habe die Hoffnung, dass ... ].

Autorka zdaje sobie sprawę, że zarówno opracowanie poprzedzajace teksty, jak $i$ aparat naukowy pracy nie moga rościć sobie praw do przedstawienia petnego obrazu życia emigracyjnego Krystyna Lacha Szyrmy, a wiele z postaci $i$ wydarzeń, o których wspomina Lach nadal wymaga zbadania. Mimo to można żywić nadzieje, że edycja wypetnia choć po części lukę w dotychczasowych badaniach nad biografia ,, syna ziemi mazurskiej" [...] [... man kann die Hoffnung hegen, dass ....].

Ważne wydaje się również krótkie omówienia gatunku, jakim jest satyra. Satyra należy do gatunków dydaktycznych, wywodzacych się z antyku [Wichtig scheint ...].

Ad. 3. Bis zu welchem Grad auch immer das wissenschaftliche Schreiben an die universellen Stilmaximen wie Objektivität, Sachlichkeit oder Klarheit (vgl. u.a. Weinrich 1989) gebunden sein mag, es ist zugleich in den jeweiligen Kommunikationsgemeinschaften und deren Konventionen verankert und daher stets einzelsprachlich bedingt. Eine auf alle Sprach- und Kulturräume applizierbare Wissenschaftssprache gibt es nicht (vgl. Kaiser 2002:20). Sicherlich gibt es innerhalb einer Sprachgemeinschaft auch individuelle wissenschaftliche Schreibstile (letztlich schreibt ja jeder Mensch anders); diese weichen aber nur bis zu einem gewissen Maße von dem konventionalisierten wissenschaftlichen Standardsprachgebrauch ab und sind stets auf die in der jeweiligen Sprach- oder Kulturgemeinschaft etablierten Diskurstraditionen bezogen. Generell gilt, dass die Wissenschaftssprache, die innerhalb einer Sprachgemeinschaft tradiert wird, ein in sich geschlossenes, relativ homogenes Konstrukt ist. Schreibt man in einer fremden Sprache, so ist man naturgemäß dazu gezwungen, die innerhalb der ursprünglichen Sprachgemeinschaft meist unreflektiert erworbenen Schreibkonventionen an die Schreibregeln in der Zielsprache anzupassen. Mithin kommt es zu einer gewissen Vermengung zweier Sichtperspektiven, ja Denkstile, der eigenen und der fremden, und in dessen Folge auch zur Kontamination unterschiedlicher Argumentationsstile und Schreibpräferenzen. Je unerfahrener der Schreiber ist, umso auffälliger sind die Idiosynkrasien in seinem Schreib- und Denkstil. Gerade im Falle von Arbeiten studentischer Schreibnovizen, die ihre Schreibpräferenzen auf die fremdsprachlichen Textstrukturen unbewusst übertragen, lässt sich ein Aufeinandertreffen von unterschiedlichen, ja inkompatib- 
len Schreibstilen beobachten. Ist man noch nicht in der Lage, sich von dem aus der Sekundärliteratur zitierten Text völlig zu lösen, kopiert man den Stil und die Darstellungsweise des referierten Autors. Auch wenn es unmöglich ist, die Grenze zwischen dem eigenen und dem fremden „Schreibparadigma“ exakt festzulegen, so lässt sich doch sprachintuitiv feststellen, was an dem jeweiligen Textteil eigen und was fremd ist. Die so entstandenen ,hybriden“ Formulierungsmuster können auf der Folie der prototypischen Wissenschaftstexte, welche sich durch eine maximale Ausgeprägtheit der domänentypischen Merkmale kennzeichnen, erkannt und interpretiert werden. Als wissenschaftlich prototypische Texte wurden hier ausgewählte Kapitel aus den Expertentexten herangezogen. Der erste oberflächliche Vergleich zeigt, dass in Arbeiten polnischer Schreibnovizen vielfach ein idiosynkratischer Schreibstil vorliegt, der sich gewissermaßen aus unterschiedlichen Schreibmustern speist, und im Endeffekt keinem der beiden Schreibparadigmen als sein (proto-)typisches Qualitätsmerkmal zugeordnet werden kann. Was nun die Verwendung der modalisierenden Signale im Besonderen angeht, so ergeben sich auf Grund der Korpusuntersuchungen folgende drei Tendenzen: Zum einen kann beobachtet werden, dass die polnischen Germanistikstudenten ähnlich wie ihre Kommilitonen aus dem Fach Polonistik relativ häufig dazu neigen, ihre Präsenz und subjektiven Meinungen explizit zu kennzeichnen. Die deutschen Studenten, verhalten sich in dieser Hinsicht eher zurückhaltend, vor allem was die Markierung der eigenen Subjektivität betrifft. Das auffälligste Merkmal dieses Vorgehens zeigt sich im Bestreben, als Schreibende auf keinen Fall in Erscheinung zu treten, wodurch die Texte unpersönlich bzw. distanziert wirken (vgl. Kaiser 2002:14). Im Gegensatz dazu sind Arbeiten polnischer Germanistikstudenten persönlicher und subjektiver. Eine Polarität liegt hier jedoch nicht vor, zumal wenn man bedenkt, dass es unter den germanistischen Arbeiten polnischer Studenten auch viele Texte gibt, die keinerlei modalisierende Ausdrücke enthalten. Der zweite, kulturspezifische Unterschied betrifft den Argumentationsstil. Während die deutschen (sowohl erfahrenen als auch wenig fortgestrittenen) Autoren die Tendenz dazu zeigen, die Richtigkeit der präsentierten Argumente durch eine Reihe von Evidenzen und Fakten sowie die Bezugnahme auf Autoritäten ${ }^{9}$ abzusichern, wird die „Stringenz“ der Argumentation in Arbeiten polnischer Schreiber durch den Bezug auf subjektive Erfahrungen bzw. Eindrücke untermauert. Daraus resultiert womöglich der quantitative Unterschied hinsichtlich der Verwendung von subjektiven Meinungsausdrücken. Drittens lässt sich in den untersuchten Studententexten eine Tendenz zur affektiven Stilhaltung aufspüren, was ebenfalls den Einsatz von modalisierenden Ausdrücken begünstigt. Die Sachverhalte werden zuweilen in einem auf Anschaulichkeit ausgerichteten, überschwänglichen, ja nahezu gefühlsbetonten

\footnotetext{
9 Vgl. dazu Kaiser (2002:178): „,das Kriterium der Nachprüfbarkeit und die Bezugnahme auf Autoritäten [ist] in den deutschen wissenschaftlichen Diskurstraditionen besonders ausgeprägt“"
} 
Stil mitgeteilt. Auch die Beschreibungen der eigenen Meinung erstrecken sich manchmal über mehrere Syntagmen. Demgegenüber sind Stellungnahmen in Arbeiten deutscher Autoren meist auf einen einfachen Ausdruck reduziert.

\section{Zusammenfassung und Ausblick}

Die Strategie der Modalisierung gilt als eine der wichtigsten wissenschaftstypischen Handlungen. Anders als bei rein evidentiell-quotativen Geltungsbegründungen von Aussagen, wo der Geltungsanspruch des Sachverhalts mit Verweis auf eine fremde (wissenschaftliche) Quelle gesichert ist, handelt es sich bei epistemisch-subjektiven Signalen um Reflexe des kritischen Denkens. Mit der Einschaltung eines jeden sprecherbasierten Kommentars, welcher Art und Komplexität auch immer, verlässt der Autor die sachliche Inhaltsebene und baut in den Textfluss evaluative Metainformationen ein. Der Gesamttext erhält so ein Relief von referierenden (darstellenden) und modalisierenden (perspektivierenden) Textteilen.

Die Strategien zur Markierung von subjektiv-modalen Urteilen kommen in den studentischen Elaboraten in unterschiedlicher Ausprägung zum Vorschein, wobei nicht alle Modalisierungssignale gemäß den wissenschaftskommunikativen Anforderungen verwendet werden, was seinerseits beweist, dass die Aneignung der wissenschaftlichen Textkompetenz in einer Fremdsprache etwas mehr als etwa bloße Verwendung der „richtigen“ Wörter oder Termini bedeutet (vgl. Ehlich 1993:34ff.).

Die Defizite bei dem Erwerb wissenschaftlicher Textkompetenzen lassen sich sehr gut anhand der gewonnenen Korpusdaten verfolgen, die konkrete sprachliche und kognitive Probleme beim Gebrauch von modalisierenden Strategien offenbaren (vgl. die Fehleranalyse im Anhang). Diese Schwierigkeiten treten zwar beim Gebrauch von Meinungsausdrücken auf, betreffen aber zugleich andere Kompetenzbereiche des wissenschaftlichen Schreibens wie etwa argumentieren, begründen, umformulieren, schlussfolgern, sich distanzieren etc. Im didaktischen Prozess sollen deshalb entsprechende Anstrengungen unternommen werden, um die Defizite möglichst umfassend zu beseitigen. Dazu benötigt man konkrete didaktische Maßnahmen, die sowohl die schreibprozessbezogenen (z.B. Schreibstrategien, Formulierungsmuster) als auch produktbezogenen (z.B. textsortentypische Merkmale, Sprachmittelrepertoire) Kompetenzen verbessern sollen. Angesichts der durchgeführten Korpusuntersuchung und im Hinblick auf die diagnostizierten Probleme können folgende didaktische Maßnahmen und Schreibaufträge vorgeschlagen werden:

- Typ 1: Dieser Typ von prozessbezogenen Schreibaufgaben umfasst alle Schreibaufträge, die darauf abzielen, den Studierenden bewusst zu machen, dass es unterschiedliche Schreibstile gibt; 
- Typ 2: Bei diesem Typ von prozessbezogenen Schreibaufgaben wird die Fähigkeit trainiert, einen Textinhalt von einer alltagssprachlichen Darstellungsweise zu einer fachlich korrekten Ausdrucksweise zu transponieren;

- Typ 3: Schreibaufgaben aus dieser Gruppe sollen die Studierenden darauf vorbereiten, eine argumentative Textstruktur aufbauen zu können;

- Typ 4: Bei diesem Typ von Schreibaufgaben handelt es sich darum, die Studierenden mit dem Phänomen der subjektiven Qualifizierungen vertraut zu machen und ihnen die Fähigkeit vermitteln, mit modalisierenden Strategien und Meinungsausdrücken wissenschaftstypisch umzugehen;

- Typ 5: Es handelt sich hierbei um Schreibaufgaben, die den Studierenden die Kompetenz vermitteln sollen, sich bewusst auf unterschiedlichen Ebenen (Sachebene vs. metakommunikative Ebene) des Textes bewegen zu können; - Typ 6: Schreibaufgaben dieses Typs zielen darauf ab, den Studierenden zum Aufbau von eigenständigen Formulierungs- und Umformulierungskompetenzen zu verhelfen;

- Typ 7: Ziel und Sinn dieser Schreibaufgaben ist, neue Ausdrucksmöglichkeiten zu entwickeln und diese mit den schon verfügbaren zu verknüpfen.

Sicherlich erhebt diese Typologisierung keinen Anspruch auf Vollständigkeit; dies wird hier auch nicht angestrebt. Die obigen Typen von Schreibaufgaben korrelieren vielmehr mit den im Untersuchungskorpus am häufigsten vorkommenden Schreibdefiziten. Ferner ist zu bemerken, dass die Lösung der Probleme hinsichtlich der Verwendung von modalisierenden Strategien ohne solide Sprachkompetenzen nicht möglich sind. Mein Plädoyer gilt daher einer frühzeitigen Gewöhnung der Studierenden an das wissenschaftliche Schreiben, damit möglichst schnell solide sprachliche Grundlagen für die eigene Weiterarbeit entwickelt werden und eine natürliche Orientierungsfähigkeit im (Wissenschafts-)Sprachlichen erworben werden kann. Damit geht das nächste dringende Desiderat einher, nämlich die Einführung von Seminaren, deren Fokus auf das Deutsche als fremde Wissenschaftssprache gerichtet werden soll. Da die germanistische Studienausbildung in ihren Grundsätzen darauf ausgerichtet ist, den Studierenden eine wissenschaftliche Selbstständigkeit zu vermitteln, deren sichtbares Zeichen das eigenständige Verfassen einer wissenschaftlichen Arbeit ist, wäre es sinnvoll, Überlegungen darüber anzustellen, inwieweit der holprige Weg zu diesem Ziel über derartige Lernveranstaltungen „eingeebnet“" werden könnte. 


\section{Literatur}

DönNinghaus Sabine, 2005, Die Vagheit der Sprache: Begriffsgeschichte und Funktionsbeschreibung anhand der tschechischen Wissenschaftssprache, Wiesbaden.

EhLICH Konrad, 1993, Deutsch als fremde Wissenschaftssprache, in: Jahrbuch Deutsch als Fremdsprache 19, S. 13-42.

EsSER Ruth, 1997, „Etwas ist mir geheim geblieben am deutschen Referat.“ Kulturelle Geprägtheit wissenschaftlicher Textproduktion und ihre Konsequenzen für den universitären Unterricht von Deutsch als Fremdsprache, München.

Gauger Hans-Martin, 1992, Das Was und das Wie. Zum Begriff des Stils, in: Gauger H.-M. (Hrsg.), Über Sprache und Stil, München, S. 208-228.

GätJe Olaf / ReZAT Sara / Steinhoff Torsten, 2012, Positionierung. Zur Entwicklung des Gebrauchs modalisierender Prozeduren in argumentativen Texten von Schülern und Studenten, in: Feilke H./Lehnen K. (Hrsg.), Schreib- und Textroutinen. Theorie, Erwerb und didaktisch-mediale Modellierung, Frankfurt am Main, S. 125-153.

Graefen Gabriele / Moll Melanie, 2011, Wissenschaftssprache Deutsch: lesen - verstehen schreiben, Ein Lehr- und Arbeitsbuch, Frankfurt am Main.

HufeISEN Britta, 2002, Ein deutsches Referat ist kein englischsprachiges Essay. Theoretische und praktische Überlegungen zu einem verbesserten textsortenbezogenen Schreibunterricht in der Fremdsprache Deutsch an der Universität, Innsbruck.

KAISER Dorothee, 2002, Wege zum wissenschaftlichen Schreiben: Eine kontrastive Untersuchung zu studentischen Texten aus Venezuela und Deutschland, Tübingen.

KotIN Michail / SснӧNHERR Monika, 2012, Zum Verhältnis von Epistemizität und Evidentialität im Deutschen aus diachroner und typologischer Sicht, in: Zeitschrift für Deutsche Philologie H. 3, S. 393-416.

KöLLER Wilhelm, 1995, Modalität als sprachliches Grundphänomen, in: Der Deutschunterricht $47 / 4$, S. $37-50$.

KöLLER Wilhelm, 2004, Perspektivität und Sprache. Zur Struktur von Objektivierungsformen in Bildern, im Denken und in der Sprache, Berlin/New York.

Kretzenbacher Heinz L., 1995, Wie durchsichtig ist die Sprache der Wissenschaften?, in: Kretzenbacher H.L./Harald W. (Hrsg.), Linguistik der Wissenschaftssprache (Forschungsbericht), Berlin/New York, S. 15-40.

OlszewsKa Danuta, 2016, Über stilistische Invarianten in wissenschaftlichen Texten auf der MetaEbene, in: Nycz K./Baumann K.-D./Kalverkämper H. (Hrsg.), Fachsprachenforschung in Polen, Berlin, S. 121-158.

SCHÖNHERR Monika, 2017, Meinungsbekundungen in akademischen Texten polnischer Studierender: Defizite, Schwierigkeiten, Optimierungsstrategien. Manuskript, Zielona Góra.

SteinHoff Torsten, 2007, Wissenschaftliche Textkompetenz: Sprachgebrauch und Schreibentwicklung in wissenschaftlichen Texten von Studenten und Experten, Tübingen.

WeINRICH Harald, 1989, Formen der Wissenschaftssprache, in: Jahrbuch 1988 der Akademie der Wissenschaften zu Berlin, Berlin, S. 119-158.

Zuchewicz Tadeusz, 2001, Befähigung zum wissenschaftlichen Schreiben in der Fremdsprache Deutsch, in: Deutsch als Fremdsprache, H. 1, S. 14-19.

Zuchewicz Tadeusz, 1997, Schreibförderungen im DaF-Studium - ein vergessenes Problem der Sprachdidaktik?, in: Materialen Deutsch als Fremdsprache, Bd. 43, S. 457-461. 


\section{The principle of objectivity in academic texts: Modalizing markers in German-Polish contrast}

Students' written works produced in an academic environment follow certain scientific conventions, such as formality or objectivity. The objective style of academic texts can be reflected in how the writer refers to the research of others or uses referential markers and modals. This paper is aimed at discussing several aspects related to objectivism and subjectivism in academic-scientific writing. Moreover, it shows the most frequent mistakes Polish students usually make in their L2 German academic texts, particularly in their bachelor and master theses.

Keywords: academic writing, L2 German academic texts, scientific objectivity, scientific text conventions, modalizing signals. 
Anhang 1: Fehleranalyse und Verbesserungsvorschläge

\begin{tabular}{|c|c|}
\hline $\begin{array}{l}\text { Signifikant ist, dass die Kognitionswissenschaft } \\
\text { als ziemlich junger Wissenschaftszweig } \\
\text { mehrdimensionale Gebiete der } \\
\text { wissenschaftlichen Disziplinen umfasst. Es } \\
\text { ist zu bedenken, dass sie die Korrelationen } \\
\text { zwischen Wahrnehmung, Denken, Motorik und } \\
\text { Sprache untersucht. }\end{array}$ & $\begin{array}{l}\text { Da der zweite Satz zu dem vorausgehenden } \\
\text { Kontext in einem explikativ-kompletiven } \\
\text { Verhältnis steht, passt hier der eher adversativ } \\
\text { markierte Meinungsausdruck nicht ganz. } \\
\text { Viel besser wäre es, auf eine modalisierende } \\
\text { Formulierung gänzlich zu verzichten, da } \\
\text { sie mit der wissenschaftlichen Prozedur der } \\
\text { Begriffsbildung (und so eine liegt auch hier } \\
\text { vor) inkompatibel sind. } \\
\text { Verbesserungsvorschlag: Signifikant ist, dass } \\
\text { die Kognitionswissenschaft als ziemlich junger } \\
\text { Wissenschaftszweig mehrdimensionale Gebiete } \\
\text { der wissenschaftlichen Disziplinen umfasst. } \\
\text { Dabei untersucht sie die Korrelation zwischen } \\
\text {... }\end{array}$ \\
\hline $\begin{array}{l}\text { Selbstverständlich ist, dass die Vergangenheit } \\
\text { nach gezielten Untersuchungsgegenständen } \\
\text { systematisiert wird. Sie kann ebenso mit } \\
\text { Hilfe des kommunikativen Gedächtnisses } \\
\text { wie des kulturellen und wissenschaftlichen } \\
\text { Gedächtnisses zum Ausdruck gebracht werden. }\end{array}$ & $\begin{array}{l}\text { Dieser Satz steht am absoluten Anfang } \\
\text { im Kapitel der Arbeit; somit liegt hier } \\
\text { kein triftiger Grund vor, die neutrale } \\
\text { Informationsstruktur des Einleitungssatzes zu } \\
\text { ändern. } \\
\text { Verbesserungsvorschlag: Es ist } \\
\text { selbstverständlich, dass die Vergangenheit ... }\end{array}$ \\
\hline $\begin{array}{l}\text { Für mich macht der Lehrer einen Eindruck, als } \\
\text { machte er sich über den Schüler lustig. }\end{array}$ & $\begin{array}{l}\text { Beide Meinungsausdrücke ähneln allzu } \\
\text { sehr dem mündlichen Sprachregister. Eine } \\
\text { andere Ausdrucksvariante, die für den } \\
\text { Wissenschaftskontext besonders typisch ist, ist } \\
\text { etwa meines Erachtens. }\end{array}$ \\
\hline $\begin{array}{l}\text { In meinen Augen liefert Tannen in ihrem Buch } \\
\text { einfache Antworten auf schwierige Fragen } \\
\text { und scheut gleichzeitig nicht vor großen } \\
\text { Generalisierungen. }\end{array}$ & $\begin{array}{l}\text { Verbesserungsvorschlag: Meines Erachtens } \\
\text { macht der Lehrer den Eindruck, als würde er } \\
\text { sich über den Schüler lustig machen. } \\
\text { Meines Erachtens liefert Tannen in ihrem Buch } \\
\text { einfache Antworten ... }\end{array}$ \\
\hline
\end{tabular}


In Brockhaus Enzyklopädie [1967:484] steht, dass alle Menschen (auch Kinder und Geisteskranke) eine Fähigkeit besitzen, sich zu beleidigen. Ich stimme damit überein, obwohl jeder auf eigene Art beleidigt und beleidigt wird.
Die Formulierungsmuster sind stark vom schulischen Schreibstil geprägt. Der Versuch, den aus dem Quelltext (der Brockhaus Enzyklopädie) geschöpften Sachverhalt mit eigenen Worten wiederzugeben, scheint hier nicht allzu gelungen zu sein. Was den Meinungsausdruck im Besonderen angeht, so wirkt er überflüssig, da die signalisierte Position des Autors weder erforderlich noch zweckmäßig ist, und angesichts des Zitats aus einem Lexikon wie der Brockhaus Enzyklopädie wohl kaum interessant. Viel wichtiger als seine subjektive Meinung ist jedoch die nachfolgend hinzugefügte Bemerkung.

Verbesserungsvorschlag: Laut der Brockhaus Enzyklopädie [1967: 484] sind alle Menschen, darunter auch Kinder und Geisteskranke, fähig, Beleidigungen auszusprechen und sich beleidigt zu fühlen, auch wenn jeder auf seine eigene Art beleidigt und beleidigt werden kann.

Meiner Meinung nach zeigen diese Beispiele, wie große Rolle Mimik des Gesichts spielt. Sie kann sowohl positive als auch negative Eindrücke machen.

Im Grunde genommen lassen sich hier keine gravierenden Verstöße gegen den studentischen Schreibusus feststellen. Der Text könnte aber sprachlich noch mehr wissenschaftlich ausgerichtet werden, indem der Meinungsausdruck durch einen anderen ersetzt und topologisch umgestellt wird.

Verbesserungsvorschlag: Allein diese wenigen Beispiele zeugen meines Erachtens davon, dass die Mimik des Gesichts hierbei eine große Rolle spielt.

Ein rundum misslungener Text; es wimmelt hier von stilistischen Unzulänglichkeiten und grammatischen sowie orthographischen Fehlern. Der Meinungsausdruck wird ungünstig in das Satzinnere eingeschoben, wodurch die Rezeption des Gesamtsatzes gestört wird.

Verbesserungsvorschlag: Ich habe dieses Thema aus dem Grunde/deswegen ausgewählt, weil es mir interessant scheint. Komplimente hört man gerne. In meiner Arbeit werde ich darstellen, wie man Komplimente sprachlich kommuniziert. Da es ein relativ umfangreiches Thema ist, konzentriere ich mich nur auf einige ausgewählte Prinzipien, die aus meiner Sicht/ meiner Meinung nach/meines Erachtens am wichtigsten sind. 
Das Thema „Geschlechtstypisches

Kommunikationsverhaltens" erfreut sich nach meiner Auffassung seit vielen Jahren eines recht großen Interesses.
Der Meinungsausdruck wird hier inkompetent verwendet, da ob das behandelte Thema sich einer großen Beachtung in der Forschung erfreut oder nicht, ist nicht die Frage der subjektiven Wertung des Schreibers, sondern die der konkreten wissenschaftlichen Fakten, die entweder dafür oder dagegen sprechen.

Verbesserungsvorschlag: Das Thema [...] erfreut sich seit vielen Jahren eines recht großen Interesses. 